\title{
Design and Evaluation of a Haptic Interface With Octopod Kinematics
}

\author{
Markus Kühne ${ }^{(0)}$, Johannes Potzy, Roberto García-Rochín, Patrick van der Smagt, and Angelika Peer
}

\begin{abstract}
The study of human motor control using functional magnetic resonance imaging gives rise to many challenges. One of them is the design of haptic interfaces that are compatible with the magnetic field. To achieve this, the existing haptic interfaces employ parallel kinematics. However, they are limited to three degrees of freedom (DOFs). When trying to offer more DOF without floating actuators, parallel kinematics suffer from direct kinematic singularities, and thus, strong mechanical anisotropy. In this paper, we determine an optimal six DOF kinematics that overcomes these limitations. To this end, we use performance indices such as singularity occurrence, worst case output capabilities, sensitivity, and the global isotropy index. The resulting Octopod kinematics avoids a range of direct kinematic singularities by design. Finally, we present and evaluate a non-magnetic-resonance-compatible prototype of this novel type of kinematics.
\end{abstract}

Index Terms-Haptic interface, parallel kinematics, redundant kinematics, singularities.

\section{INTRODUCTION}

$\mathbf{P}$ ARALLEL kinematics have been employed as haptic interfaces to carry out human motor control studies using functional magnetic resonance imaging (fMRI) [1]-[3]. Due to their nonfloating actuators, they are less likely to cause image artifacts than serial kinematics. Moreover, they provide a high stiffness, a small inertia as well as a high force bandwidth. However, for more than three degrees of freedom (DOFs), parallel kinematics are likely to be subject to both direct and inverse

Manuscript received April 14, 2016; revised July 29, 2016, March 24, 2017, and July 18, 2017; accepted August 9, 2017. Date of publication August 20, 2017; date of current version October 13, 2017. Recommended by Technical Editor X. Chen. This work was supported in part by the German Research Foundation (DFG) within the VR System for Visuo-Haptic Stimulation in the Context of fMRI Analyses project. (Corresponding author: Markus Kühne.)

M. Kühne is with the Bristol Robotics Laboratory, University of the West of England, Bristol BS16 1QY, U.K., and also with the Biomimetic Robotics and Machine Learning Laboratory, Technische Universität München, Munich 80333, Germany (e-mail: Markus.Kuehne@tum.de).

J. Potzy is with the Biomimetic Robotics and Machine Learning Laboratory, Technische Universität München, Munich 80333, Germany (e-mail: Johannes.Potzy@googlemail.com).

R. García-Rochín is with the Tecnologico de Monterrey, Escuela de Ingeniería y Ciencias, Monterrey, N.L. 64849, México (e-mail: rober_ rgr@ hotmail.com).

P. van der Smagt is with the Volkswagen Group, Germany (e-mail: smagt@brml.org)

A. Peer is with the Bristol Robotics Laboratory, University of the West of England, Bristol BS16 1QY, U.K. (e-mail: Angelika.Peer@brl.ac.uk).

Color versions of one or more of the figures in this paper are available online at http://ieeexplore.ieee.org.

Digital Object Identifier 10.1109/TMECH.2017.2742581 kinematic singularities. Direct kinematic singularities result in the gain of an uncontrollable DOF, while inverse kinematic singularities lead to the loss of one DOF [4]. If these singularities occur inside the desired workspace, they render the parallel kinematics unusable as haptic interfaces. Besides the kinematic structure, the actuation principle plays an important role for magnetic resonance (MR)-compatibility. The haptic devices are actuated by shielded direct current (dc) motors [2] or by rotary traveling wave ultrasonic motors (USMs) [1], [3]. Advantages of USMs over linear actuation possibilities, such as pneumatic or hydraulic pistons, have been discussed by Klare et al. [1]. However, the position control of USMs remains challenging [5]. Hence, designing an MR-compatible haptic interface using USMs consists of two separate tasks: 1) development of a position controller for USMs; and 2) designing a parallel kinematics. In this paper, we focus on the latter challenge with specific focus on designing a kinematics with six DOF.

In literature a range of kinematics have been proposed. Klare et al. [1] has adopted the well-known Delta kinematics for the design of an MR-compatible haptic interface. This type of kinematics provides three translational DOF and is not subject to direct kinematics singularities. However, the operator has restricted natural motions, since he cannot rotate the end effector. Pierrot et al. [6] showed that the HEXA, which uses a fullyparallel kinematics, is a straightforward extension to the Delta robot providing six DOF. Criteria for his design were maintaining nonfloating rotational actuators, high dynamic capabilities with six DOF, and simplicity. The HEXA has six identical legs, each one actuated by a rotary actuator and composed of two spherical joints. These so-called RSS legs are arranged in pairs. When each pair moves simultaneously, the HEXA operates like the Delta only in three translational DOF. Due to the benefits of this type of kinematics with six DOF, a range of variants have been used as prototypical haptic interfaces in [7]-[9]. However, direct kinematic singularities occur and restrict the workspace.

Commercial haptic interfaces, on the other hand, increase the singularity-free workspace of kinematics with six DOF by employing hybrid parallel-serial architectures. For example, the omega. 6 or sigma. 7 from force dimension are based on Delta kinematics with three translational DOF. Actuators at the end effector are in series with the Delta kinematics and add three rotational DOF. This comes at the cost of reduced output capabilities and increased inertia [10]. Moreover, MR-compatibility issues are likely to arise since floating actuators are employed.

In this paper, we propose a parallel kinematics that provides six DOF, avoids singularities by design, and does not employ 
floating actuators. The kinematics is driven by rotary actuators such that it can be built using MR-compatible USMs. Thus, the main contribution of this paper is a novel Octopod kinematics that is systematically developed by comparing existing Hexapods based on quantitative performance criteria. These criteria include singularity occurrence, isotropy, sensitivity, as well as worst case output capabilities. To this end, also a comprehensive algorithm to determine maximum sensitivity and worst case output capabilities of actuation-redundant parallel kinematics is introduced. Finally, the kinematics is realized as a non-MRcompatible prototype. This allows the study of the kinematics independently from position control issues of USMs.

This paper is structured as follows. Section II specifies the design criteria and presents the selection procedure for the haptic interface. The kinematics selection and design optimization are described in Section III. The results are discussed in Section IV and conclusions are drawn in Section V.

\section{Design Criteria ANd Procedure, AND PERFORMANCE INDICES}

This section presents the design criteria that should be met by the kinematics. It also outlines the procedure to fulfill these criteria. Moreover, the performance indices that were applied within the procedure are introduced.

\section{A. Design Criteria}

The goal of this paper is to develop a haptic interface that fulfills the following design criteria.

1) Six actuated DOF that enable the operator to perform natural, unconstrained movements: $d=6$.

2) Rotary, nonfloating actuators directly at the joints that allow obtaining a parallel kinematics that can be built using USMs. Hence, only the first joint of each leg should be actuated and the number of legs $L$ has to be at least as big as the number of DOF: $L \geq d$.

3) The minimum output capabilities should be $1 \mathrm{~m} / \mathrm{s}$ for the translational velocity and $9.81 \mathrm{~m} / \mathrm{s}^{2}$ for the translational acceleration to provide a natural feeling as specified by Fisher and Daniel [11]

4) The dexterous workspace should be free of singularities, have a minimum volume of $150 \mathrm{~mm} \times 150 \mathrm{~mm} \times$ $150 \mathrm{~mm}$ and provide $\pm 30^{\circ}$ of rotational DOF around all axes of the end effector [11]. This allows natural reaching motions including arm transport.

5) The mechanical isotropy should be optimized to render a virtual environment with the same fidelity in the entire workspace.

6) The maximum sensitivity of the device should be quantifiable in terms of force, torque, position, and orientation, in order to estimate worst case error amplification.

\section{B. Adopted Procedure for Kinematic Selection and Design Optimization}

In order to meet our design criteria, the kinematics is selected and optimized using the following four step procedure.
In the first step, existing fully-parallel kinematics with rotary actuators are selected. Then, a parametrized description of these kinematics is defined to be able to compare them systematically.

In the second step, we determine a kinematics among the existing configurations that is optimal regarding our design criteria quantified by means of performance indices. Several indices have been introduced to determine the kinematic and dynamic performance of parallel manipulators as well as haptic interfaces [7], [12]-[15]. Here, we focus on performance indices that allow meeting our design criteria: Worst case output capabilities to guarantee required minimum output capabilities, singularity occurrence to avoid singularities within the dexterous workspace as well as the global isotropy index (GII) [12] to provide mechanical isotropy. Moreover, the sensitivity is evaluated in order to quantify maximum error amplification. Finally, the size of the dexterous workspace is calculated such that it guarantees the minimum required volume.

In the third step, the optimal kinematics resulting from the selection is enhanced systematically. Kinematic or actuation redundancy can be employed to further reduce singularity occurrence [4]. This also enhances isotropy as well as output capabilities as will be shown in this paper. Redundancy can also be employed to provide additional DOF, e.g., for grasping [16]. Kinematic redundancy means that at least one linkage is a motion generator of more DOF than necessary. Actuation redundancy means that the number of DOF is overconstrained by the number of actuators and is again split up in two classes: In-branch redundancy, where at least one leg has more actuated DOF than necessary; and branch redundancy, where the end effector is overconstrained by at least one kinematic chain. In-branch redundancy requires floating actuators or has to be realized via linkages from additional motors to the joints that should be actuated. This results in quasi-in-branch redundant kinematics [9], but requires complex kinematic chains. Here, we suggest a branch-redundant solution. It enlarges the singularityfree workspace and maintains the simplicity of the nonredundant kinematics. An additional pair of legs overconstrains the end effector of a Hexapod and results in an Octopod configuration.

In the fourth and final step, a mechatronic prototype of the kinematics is realized. To achieve the desired performance, also backlash, friction, and mass have to be minimized, which is done during the mechanical design process of this work. The prototype is then employed to validate the kinematics.

\section{Performance Indices}

The performance indices employed in this paper and used for selection as well as optimization of the kinematics mostly rely on the Jacobian matrix. For parallel manipulators, the Jacobian is often decomposed and yields the relation between actuator velocities $\dot{\mathbf{q}}$ and output velocities $\dot{\mathbf{x}}$ as

$$
\mathbf{J}_{\mathbf{q}} \dot{\mathbf{q}}=\mathbf{J}_{\mathbf{x}} \dot{\mathbf{x}}
$$

Here, $\mathbf{J}_{\mathbf{q}}$ is a square $L \times L$ matrix and $\mathbf{J}_{\mathbf{x}}$ is a $L \times d$ matrix, where $d$ is the number of DOF of the device and $L$ is the number of legs and actuators. $\mathbf{J}_{\mathbf{x}}$ is square for nonredundant actuation. The Jacobian for parallel manipulators is then typically 
expressed as

$$
\mathbf{J}=\mathbf{J}_{q}^{-1} \mathbf{J}_{x}
$$

and the force/torque output capabilities are denoted by

$$
\mathbf{f}=\mathbf{J}^{T} \boldsymbol{\tau} \text {. }
$$

This establishes the relation between the actuator torques $\tau$ and the end effector wrench $\mathrm{f}$. Note that this definition is different from serial manipulators. The Jacobian can be employed to measure closeness to singularities [17], isotropy [12], as well as output capabilities of the device such as forces, torques, or velocities [18].

1) Kinematic Singularities: The major problem of parallel kinematics with more than three DOF are kinematic singularities. In many applications singularity-free trajectories are computed for the end effector. But in case of a haptic interface, the operator can drive the kinematics into a singular configuration. Mathematically, inverse kinematic singularities occur when $\mathbf{J}_{\mathbf{q}}$ drops rank, while direct kinematic singularities are present when $\mathbf{J}_{\mathbf{x}}$ drops rank. Typical inverse kinematics singularities are found at the border of the workspace and thus, can be avoided by restricting the workspace. However, this does not guarantee avoiding all singularities of this type. Direct kinematic singularities result in the gain of an uncontrollable DOF and are often found within the workspace. In this paper, we use the word "singularity" to refer exclusively to direct kinematic singularities.

Singularity occurrence can be explained geometrically using Grassman geometry [19]. The closeness to singularities can be measured by singular values of $\mathbf{J}_{\mathbf{x}}$ or $\mathbf{J}$, respectively [17]. This is computationally easier than computing geometric configurations leading to a singularity. However, the workspace has to be sampled very precisely during the design phase to detect as many singular configurations as possible. Methods like the interval-based analysis [20] circumvent this issue by using branch-and-prune algorithms. In this study, we employ the CuikSuite toolbox [21] to detect singularities. The toolbox uses a branch-and-bound algorithm and is geared toward singularity detection in closed kinematic chains. Still, we would like to emphasize that this is not a formal proof since any numerical software tool has tolerances, which have to be taken into account.

2) Isotropy: The Jacobian can also be employed as a measure of isotropy or manipulability. Mechanical isotropy provides homogeneous output capabilities in the entire workspace. It facilitates actuator choices as well as controller design. Global isotropy makes it possible to render physical contacts with the same fidelity at every point in the workspace for every end effector pose. Manipulability or isotropy are determined locally by the condition number $\kappa$, which is given by

$$
\kappa=\frac{\sigma_{\min }}{\sigma_{\max }}
$$

where $\sigma_{\min }$ and $\sigma_{\max }$ are the minimum and maximum singular values in a specific pose. This index is expanded to the entire workspace by applying the GII. The GII characterizes global mechanical isotropy of a kinematics and has been introduced by Stocco et al. [12]. It describes the ratio of minimum and maximum singular values of the Jacobian $\sigma_{\min }$ and $\sigma_{\max }$ in the entire workspace. This index is bounded between zero and one. A GII of one represents a kinematics that is perfectly isotropic with the same output capabilities in every pose. A GII close to zero stands for a kinematics that has at least one singular configuration within its workspace. Here, a GII optimization, using the culling algorithm [12], is applied to determine the kinematics with the best isotropy from a parametrized description of the known manipulators, as depicted in Fig. 2. This allows the determination of the kinematics with best isotropy, given a certain parameterization, even if it is a hybrid solution of the known kinematics. To account for nonhomogeneous physical units in the Jacobian, a scaling matrix as proposed by Stocco et al. [22] is employed.

3) Worst Case Output Capabilities: Hayward and Astley [15] suggest to identify, besides other criteria, the best and the worst case output capabilities of haptic interfaces. They encompass force, torque, velocity, and acceleration. This allows us to determine if a kinematics can achieve the desired minimum velocity and acceleration capabilities. Ueberle [18] introduced an algorithm to evaluate the worst case output capabilities of nonredundant manipulators. Here, we propose an extension to the case of actuation-redundant kinematics. This is required, when analyzing the proposed Octopod kinematics. First of all, the equations of the output capabilities of a parallel manipulator are given by

$$
\begin{aligned}
\dot{\mathbf{x}} & =\mathbf{J}^{\#} \dot{\mathbf{q}} \\
\mathbf{f} & =\mathbf{J}^{T} \boldsymbol{\tau} ; \dot{\mathbf{q}}=\mathbf{0} \\
\ddot{\mathbf{x}} & =\mathbf{J}^{\#} \mathbf{M}_{q}(\mathbf{q})^{-1} \boldsymbol{\tau} ; \dot{\mathbf{q}}, \mathbf{f}=\mathbf{0}
\end{aligned}
$$

where $\mathbf{J}^{\#}$ is the generalized Moore-Penrose pseudoinverse, defined as $\mathbf{J}^{\#}=\left(\mathbf{J}^{T} \mathbf{J}\right)^{-1} \mathbf{J}^{T}$. These equations relate actuator capabilities, such as velocities $\dot{\mathbf{q}}$ and torques $\tau$, to end effector capabilities, namely its velocities $\dot{\mathbf{x}}$, its wrench $\mathbf{f}$, and its accelerations $\ddot{\mathbf{x}}$. The relations build on the Jacobian $\mathbf{J}$ and the mass matrix in joint space $\mathbf{M}_{q}(\mathbf{q})$. All relations can be generally written as

$$
\left(\begin{array}{l}
\mathbf{u}_{\text {trans }} \\
\mathbf{u}_{\text {rot }}
\end{array}\right)=\left(\begin{array}{l}
\hat{\mathbf{J}}_{\text {trans }} \\
\hat{\mathbf{J}}_{\text {rot }}
\end{array}\right) \mathbf{p}
$$

where $\hat{\mathbf{J}}_{\text {trans }}$ and $\hat{\mathbf{J}}_{\text {rot }}$ are the submatrices denoting the translational and the rotational part of $\hat{\mathbf{J}}$. The actuator torques and velocities are expressed by $\mathbf{p}$ and the output capabilities by $\mathbf{u}_{\text {trans }}$ as well as $\mathbf{u}_{\text {rot }}$. The worst case output capabilities are then formulated as the minimax optimization problem

$$
\min _{\mathbf{d}} \max _{\alpha>0}\|\hat{\mathbf{J}} \alpha \mathbf{d}\| \text { such that } \alpha \mathbf{d} \in\left[\mathbf{p}_{\min }, \mathbf{p}_{\max }\right]
$$

where $\|\cdot\|$ denotes the $L_{2}$ norm and $\mathbf{p}_{\min }$ and $\mathbf{p}_{\max }$ are the minimum and maximum output capabilities of the actuators. Ueberle [18] shows that the problem can be converted into six linear least squares problems for the nonredundant case, $L=$ $d$. This holds true in this situation since the minimum of the maximum achievable outputs of the end effector are found on hyperplanes, each one defined by at least one saturated actuator 
$i$, such that

$$
\begin{aligned}
& \min _{i}\left\{\min _{\mathbf{p}_{i}} \mathbf{F}_{i}^{T} \mathbf{F}_{i}\right\} \\
& \mathbf{F}_{i}=\hat{\mathbf{J}}_{i} \mathbf{p}_{i}+\hat{\mathbf{j}}_{i} \bar{p}_{i}
\end{aligned}
$$

where $\hat{\mathbf{J}}_{i}$ is obtained by deleting the $i$ th column $\hat{\mathbf{j}}_{i}$ of $\hat{\mathbf{J}}$, and $\bar{p}_{i}$ is $p_{\min }$ or $p_{\max }$. Additional constraints such as

$$
\min _{i}\left\{\min _{\mathbf{p}_{i}} \mathbf{F}_{i}^{T} \mathbf{F}_{i}\right\} \text { such that } \hat{\mathbf{J}}_{\text {rot }, \mathrm{i}} \mathbf{p}_{i}+\hat{\mathbf{j}}_{\text {rot }, \mathrm{i}} \bar{p}_{i}=0
$$

can be included in a straightforward manner [18]. Here, $\hat{\mathbf{j}}_{i}$ is the $i$ th column of the Jacobian matrix and $\bar{p}_{i}$ is the maximum output of the $i$ th actuator in terms of torque or velocity. This allows evaluating the worst case output capabilities, e.g., for pure force at zero torque output. However, in case of actuation-redundant kinematics, the minimax optimization problem (9) cannot be converted into a linear least squares problem. The additional actuators lead to infinite many solutions of (10) and (11) with a minimum output capability of zero.

In this paper, we present an algorithm that allows obtaining the worst case output capabilities of actuation-redundant kinematics. In case of nonredundant kinematics, it yields the same results as (10) and (11). To this end, we combine the work by Ueberle [18] with another approach by Krut et al. [23], who introduced an algorithm that computes a wrench polytope in task space, in order to find the "maximum operational isotropic force." The polytope in task space can be determined for both redundant and nonredundant kinematics, since each of its vertices is defined by all actuators saturating at their minimum or maximum output capability $\bar{p}_{\text {min }}$ or $\bar{p}_{\text {max }}$, respectively. Hence, all vertices and thus, all hyperplanes that constitute the polytope can be computed. The shortest distance from the origin to the surface of the polytope in task space then constitutes the worst case output capabilities. Krut et al. [23] determined the worst case wrench. In addition, we also compute worst case velocities or accelerations by considering the equations used by Ueberle [18] (5)-(7). Also, additional constraints as in (12) such that pure translational or rotational outputs can be evaluated, are added to our computation. This yields an algorithm with four steps, which we call the "polytope algorithm" here. The algorithm requires the generalized Jacobian $\hat{\mathbf{J}}$ as well as the maximum actuator output capabilities in terms of velocity, force, and resolution $\bar{p}_{i}$ as inputs. The output of the algorithm are minimum and maximum $\left\|\mathbf{u}_{\min , \max }\right\|$ achievable capabilities in terms of force, torque, velocity, acceleration, and sensitivity at the end effector. An example in three DOF is depicted in Fig. 1.

The reduction to three DOF is carried out in order to visualize the results, but all steps also apply to kinematics with more DOF. The algorithm proceeds as follows:

a) Finding all vertices of the wrench, velocity, and acceleration polytope: First of all, the vertices of the wrench, velocity, and acceleration polytope are computed. To this end, (8) is evaluated with all $2^{L}$ binary combinations of the elements of $\mathbf{p}=\left[\bar{p}_{1} \ldots \bar{p}_{i}\right]^{T}$, where $\mathbf{p}$ is $L \times 1$ and $\bar{p}_{i}$ is $p_{\min }$ or $p_{\max }$. The result is a set $\mathcal{S}$ containing the output capability vertices $\mathbf{u}_{\text {vertex }, j}$ with $j \in 1 \ldots 2^{L}$. An example, with a two dimensional force and a one dimensional torque output, is depicted in Fig. 1(a).

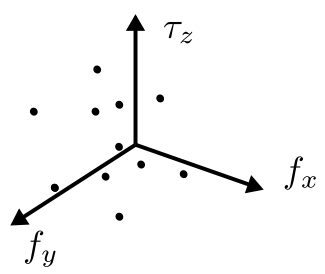

(a)

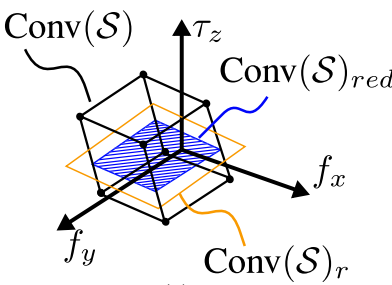

(c)

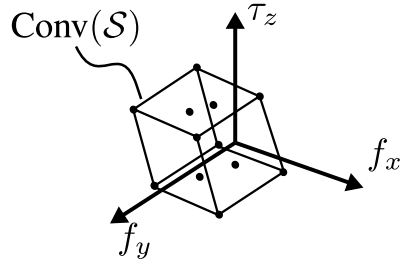

(b)

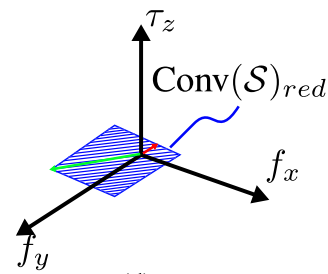

(d)
Fig. 1. Four steps (a)-(d) of the "polytope algorithm" with an example in three DOF.

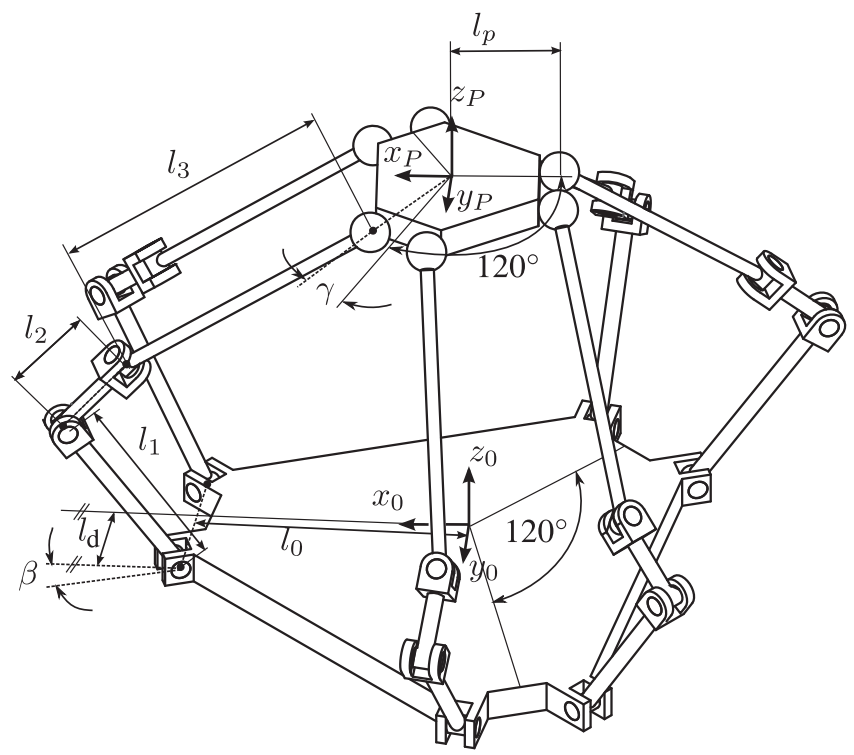

Fig. 2. General description of an RSS Hexapod, generalized as an RRRS Hexapod.

b) Determining the convex hull of the polytope: The convex hull of the polytope in $\mathbb{R}^{d}$ is described as

$$
\operatorname{Conv}(\mathcal{S})=\left\{\sum_{j=1}^{2^{L}} \alpha_{j} \mathbf{u}_{\text {vertex }, j} \mid\left(\forall j: \alpha_{j} \geq 0\right) \wedge \sum_{j=1}^{2^{L}} \alpha_{j}=1\right\} .
$$

Readily available algorithms such as quickhull are embedded in the MATLAB environment, such that (13) can be solved. The result for our example with $d=3$ is depicted in Fig. 1(b), yielding a cuboid hull.

c) Slicing the polytope along the dimensions that should be evaluated: If only output capabilities along $r$ DOF of the end effector should be evaluated, e.g., only force at zero torque output, the polytope is sliced along the DOF of interest. The result is a reduced convex hull $\operatorname{Conv}(\mathcal{S})_{\text {red }}$ in $\mathbb{R}^{r}$, with 
$0<r<d$ that is obtained via

$$
\operatorname{Conv}(\mathcal{S})_{\text {red }}=\operatorname{Conv}(\mathcal{S}) \cap \operatorname{Conv}(\mathcal{S})_{r}
$$

where $\operatorname{Conv}(\mathcal{S})_{r} \in \mathbb{R}^{r}$ is infinitely large. In the example depicted in Fig. 1(c), the polytope is sliced along the $x y$ plane, such that $r=2$. The result is the shaded blue area, in order to evaluate pure force at zero torque output.

d) Computing the minimum distance from the origin to the surface of the polytope: In the final step, the minimum distance from the origin $\mathbf{x}_{0}$ to the surface of the reduced convex hull $\operatorname{Conv}(\mathcal{S})_{\text {red }}$ is computed. In Fig. 1(d) this is indicated by a red arrow. Using the minimum distance

$$
\left\|\mathbf{u}_{\mathrm{min}}\right\|=\min _{\mathbf{c} \in \operatorname{Conv}(S)_{\mathrm{red}}}\left\|\mathbf{c}-\mathbf{x}_{0}\right\|
$$

worst case output capabilities are quantified.

4) Maximum Sensitivities: Maximum point-displacement and rotation sensitivity has been introduced by Cardou et al. [24] and can also be determined with the "polytope algorithm." This requires using (5) and considering $\dot{\mathbf{q}}$ as normalized actuator displacements, such that $\|\dot{\mathbf{q}}\|_{\infty}=\|\mathbf{J} \mathbf{x}\|_{\infty}=1 \mathrm{rad}$. Force and torque sensitivities are determined analogously using (6) and $\|\mathbf{f}\|_{\infty}=\left\|\mathbf{J}^{T} \boldsymbol{\tau}\right\|_{\infty}=1 \mathrm{~N} \cdot \mathrm{m}$. This means the actuator output capabilities are normalized to $\bar{p}_{i}= \pm 1 \mathrm{rad}$ and $\bar{p}_{i}= \pm 1 \mathrm{~N} \cdot \mathrm{m}$. The algorithm is carried out similarly and in its last step, with the maximum distance

$$
\left\|\mathbf{u}_{\max }\right\|=\max _{\mathbf{c} \in \operatorname{Conv}(S)_{\text {red }}}\left\|\mathbf{c}-\mathbf{x}_{0}\right\|
$$

maximum sensitivities are quantified. In Fig. 1(d) this is indicated by a green arrow.

5) Dexterous Workspace: Improved output capabilities can be achieved at the cost of a reduced dexterous workspace: The workspace could be reduced to a small entity with optimal output capabilities. Hence, the dexterous workspace should be taken into account as a performance index. It denotes the volume $V$ in which the end effector can achieve orientations within a specified range [4] and minimum output capabilities can be provided such that

$$
\forall \mathbf{x} \in V:|\dot{\mathbf{x}}(\mathbf{x})| \geq \dot{x}_{\min },|\ddot{\mathbf{x}}(\mathbf{x})| \geq \ddot{x}_{\min },|\mathbf{f}(\mathbf{x})| \geq f_{\min } .
$$

Here, $\dot{x}_{\mathrm{min}}, \ddot{x}_{\mathrm{min}}$, and $f_{\mathrm{min}}$ denote the minimum required output capabilities in terms of velocity, acceleration, and wrench, respectively. This workspace is computed from the inverse kinematics, where an over-approximation of the workspace is sampled. Then, only configurations, where the joint angles are real and in a valid range are retained.

\section{Kinematics Selection and Design Optimization}

In the following section, we apply the performance indices within our proposed four step procedure. This allows us to obtain an optimal kinematics that meets our design criteria.

\section{A. Step 1: Selection of Fully-Parallel Kinematics With Rotary Actuators}

In the first step, kinematics are selected for comparison. A wide range of parallel kinematics exists but here we focus on

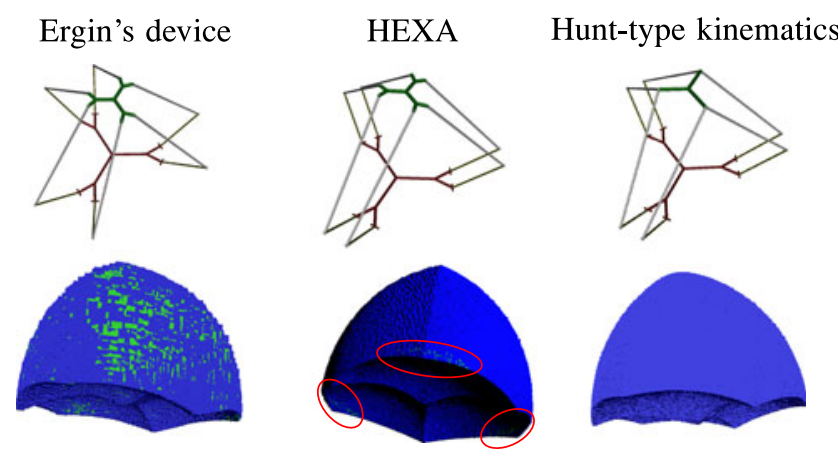

Fig. 3. Results of CuikSuite calculations for Ergin's device, the HEXA, and the Hunt-type kinematics. The dexterous workspace with only translational displacements at zero rotations is depicted in blue and singularities are depicted in green.

fully-parallel kinematics [6] with nonfloating rotational actuators. This allows the conception of an MR-compatible device actuated by USMs. The RSS kinematics meets these criteria and has been introduced by Hunt [25] already in 1983. Since the kinematics is simple and provides high dynamic capabilities [6], various variants [6]-[9] have been employed as haptic interfaces. In most cases, the original RSS chains are reduced to $\underline{R} U S$ chains, by replacing one spherical joint with a universal joint, without the loss of DOFs at the end effector. Pierrot [6] showed that his 6-RUS variant, the HEXA, is a straight-forward extension to the singularity-free Delta kinematics.

Further variants generalize the $\underline{R} U S$ chain to an $\underline{R} R R S$ chain as done by Ergin and Peer [8]. These simplifications allow the use of rotary joints only and increase the workspace [8]. A general description of a 6-ㅈS manipulator is depicted in Fig. 2. Three parameters make the difference between the Hunt-type kinematics, Ergin's device, and the HEXA: 1) angle $\beta$ at the base; 2) length $l_{2}$; and 3) angle $\gamma$ at the end effector. For the Hunt-type kinematics angles $\gamma$ and $\beta$ as well as $l_{2}$ are zero. In comparison, $\gamma$ is nonzero for the HEXA. In case of Ergin's device both $\gamma$ and $\beta$ as well as $l_{2}$ are nonzero. Among the variants of the RSS kinematics it remains unclear which one is an optimal haptic interface. Here, the HEXA [6], the iFeel6.0 [7] that implements the Hunt-type kinematics, and Ergin's device [8] were chosen for comparison.

\section{B. Step 2: Determination of an Optimal Kinematics}

In the second step, the selected kinematics were evaluated based on singularity occurrence, GII, worst case output capabilities, maximum sensitivity, and dexterous workspace.

1) Singularities: The three selected kinematics were tested for singularity occurrence in the reachable workspace. Only translational displacements at zero rotational angles were considered for illustration. Conditioning the end effector to zero rotations restricts the number of singularities that can be detected and has been done to maintain a reasonable computation time. Singularities were detected via the CuikSuite toolbox and the result is shown in Fig. 3. The Hunt-type kinematics is free of singularities in the translational workspace, unlike Ergin's device or the HEXA. Ergin's device suffers from singularities 


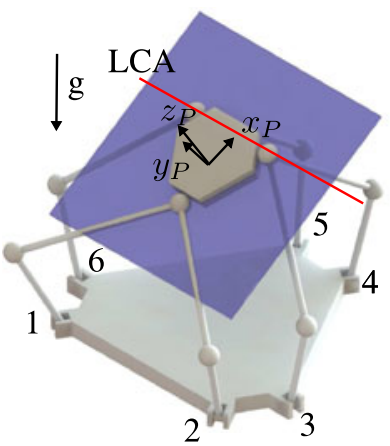

Hunt's singularity

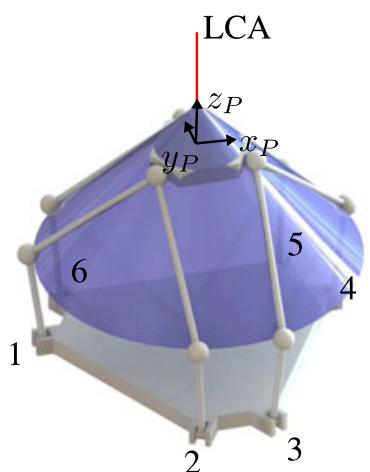

Fichter's singularity
Fig. 4. Direct kinematic singularities that are present in the workspace of an RSS Hexapod: Hunt's singularity occurs when two terminal links are coplanar with the end effector and an uncontrollable DOF around the LCA is present. In Fichter's singularity, the end effector describes an uncontrollable screw motion along the $z_{P}$ axis that aligns with the LCA.

TABLE I

Parameter Ranges of $l_{2}, \beta, \gamma$, AND $l_{d}$ FOR GII Optimization

\begin{tabular}{lcccc}
\hline \hline Parameter & Min. & Max. & Step & Optimum \\
\hline$l_{2}$ & $0 \mathrm{~mm}$ & $30 \mathrm{~mm}$ & $5 \mathrm{~mm}$ & $0 \mathrm{~mm}$ \\
$\beta$ & $0^{\circ}$ & $30^{\circ}$ & $10^{\circ}$ & $0^{\circ}$ \\
$\gamma$ & $0^{\circ}$ & $30^{\circ}$ & $10^{\circ}$ & $0^{\circ}$ \\
$l_{d}$ & $20 \mathrm{~mm}$ & $40 \mathrm{~mm}$ & $5 \mathrm{~mm}$ & $20 \mathrm{~mm}$ \\
\hline
\end{tabular}

within its entire workspace. Singularities of the HEXA occur in three distinct regions of the workspace.

Generally, singularities of Hexapods can be analyzed using Grassman geometry. Merlet has shown that singularities of parallel robots with at least six limbs occur when the lines along the limbs lie in one linear complex approximation (LCA) [19]. Two typical types of singularities of RSS Hexapods are depicted in Fig. 4. Hunt's singularity occurs when the end effector is copla-

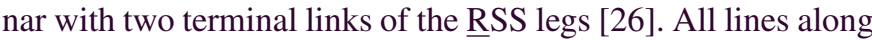
the terminal links lie in one LCA around which an uncontrolled DOF occurs. In Fichter's singularity, the end effector describes an uncontrollable screw motion along the $z_{P}$ axis that aligns with the LCA [26].

2) Isotropy: The isotropy of the known kinematics is compared via a GII optimization. Three parameters, as detailed earlier and depicted in Fig. 2, determine the difference between Hunt-type kinematics, Ergin's device, and HEXA: Angle $\beta$ at the base, length $l_{2}$, and angle $\gamma$ at the end effector. Distance $l_{d}$ was found to be another important parameter and was also considered during the optimization. The total length of each leg remains constant, which is ensured by keeping $l_{2}+l_{3}$ constant. Baseline geometric characteristics were taken from Ergin's device [8]. The step sizes were chosen as $5 \mathrm{~mm}$ for $l_{2}$ and $l_{d}$, and as $10^{\circ}$ for both $\beta$ and $\gamma$. The resulting parameter space is summarized in Table I. The step size was restricted such that it guarantees reasonable computation times. The optimization also allows hybrid solutions of the three kinematic structures. The parameters that remain constant are the length of the first
TABLE ॥

Cuboid Workspace FOR GII OPtIMIZATION CENTERED AT $z_{0}=0.30 \mathrm{M}$

\begin{tabular}{lccc}
\hline \hline \multicolumn{4}{c}{ Translational workspace } \\
Axis & Min. & Max. & Step \\
\hline$x$ & $-75 \mathrm{~mm}$ & $75 \mathrm{~mm}$ & $10 \mathrm{~mm}$ \\
$y$ & $-75 \mathrm{~mm}$ & $75 \mathrm{~mm}$ & $10 \mathrm{~mm}$ \\
$z$ & $-75 \mathrm{~mm}$ & $75 \mathrm{~mm}$ & $10 \mathrm{~mm}$ \\
\hline \multirow{4}{*}{ Rotational workspace } \\
Axis & Min. & Max. & Step \\
\hline$x$ & $-20^{\circ}$ & $20^{\circ}$ & $5^{\circ}$ \\
$y$ & $-20^{\circ}$ & $20^{\circ}$ & $5^{\circ}$ \\
$z$ & $-20^{\circ}$ & $20^{\circ}$ & $5^{\circ}$ \\
\hline \hline
\end{tabular}

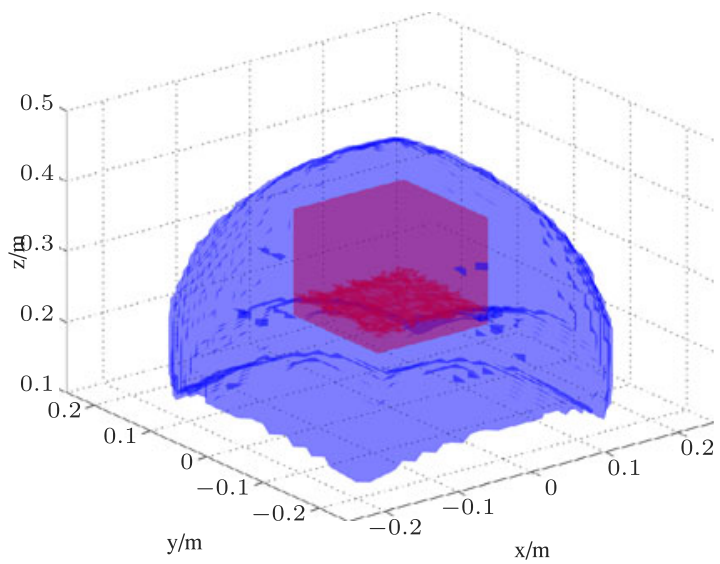

Fig. 5. Dexterous workspace, depicted with only translational displacements of the Octopod kinematics in blue and desired workspace volume, where $\pm 20^{\circ}$ rotations around all axes are achievable, in red.

link in each leg $l_{1}=157 \mathrm{~mm}$ as well as the radius of the base $l_{0}=152 \mathrm{~mm}$ and the end effector $l_{p}=55 \mathrm{~mm}$. These parameters do not contribute to the structural difference between the three kinematics to be compared. The translational workspace is a cube of $150 \mathrm{~mm}$ edge length, sampled with a step size of $10 \mathrm{~mm}$ and an offset along the $z_{0}$ axis of $0.30 \mathrm{~m}$, see Table II. The rotations around each axis of the end effector are limited to $\pm 20^{\circ}$ with a step size of $5^{\circ}$. The maximum orientations of the end effector have been restricted to $20^{\circ}$ for the optimization, since the kinematics fall into a perfect Hunt-type singularity for larger orientations within the desired dexterous workspace and hence, the minimum singular value $\sigma_{\min }$ depends only on the numerical precision and the GII is meaningless.

The optimization was computed using the culling algorithm [12]. The scaling matrix $S_{T}$ [22] was set to $S_{T}=$ $\operatorname{diag}[15 \mathrm{~N} 15 \mathrm{~N} 15 \mathrm{~N} 1 \mathrm{~N} \cdot \mathrm{m} \mathrm{1N} \cdot \mathrm{m} \mathrm{1N} \cdot \mathrm{m}]$ to account for nonhomogeneous physical units in the Jacobian. The scaling matrix is also a design choice and contains the desired magnitude of the end effector wrench. The quantities were chosen as the originally intended output capabilities for Ergin's device [8].

The optimum for the GII was found for $l_{2}=0 \mathrm{~mm}$, $\beta=0^{\circ}, \gamma=0^{\circ}$, and $d=20 \mathrm{~mm}$. Here, the GII is 0.0006 with $\sigma_{\min }=0.0127$ and $\sigma_{\max }=20.0052$. Hence, regarding the GII in the chosen parameter space and workspace, the Hunt-type 
TABLE III

Minimum and Maximum of the Worst Case Output Capabilities as Well as Maximum Sensitivities in the Workspace Listed in Table II With ROTATIONS OF $\pm 20^{\circ}$ AROUND ALL AXES

\begin{tabular}{|c|c|c|c|c|c|}
\hline \multirow[t]{2}{*}{ Output capability } & \multicolumn{5}{|c|}{ Kinematics and min. (max.) value } \\
\hline & Ergin's device & HEXA & Hunt-type & Octopod (Rot. $\pm 30^{\circ}$ ) & Octo (Rot. $\pm 30^{\circ}$ ) \\
\hline Continuous force $[\mathrm{N}]$ & $0.00(12.72)$ & $0.57(26.25)$ & $1.89(40.16)$ & $21.68(63.19)$ & $16.02(44.11)$ \\
\hline Continuous torque $[\mathrm{N} \cdot \mathrm{m}]$ & $0.00(0.59)$ & $0.03(1.17)$ & $0.05(1.75)$ & $0.86(2.80)$ & $0.75(2.14)$ \\
\hline Velocity $[\mathrm{m} / \mathrm{s}]$ & $2.78(7.85)$ & $2.78(8.01)$ & $2.86(7.98)$ & $2.74(10.06)$ & $2.93(10.18)$ \\
\hline Angular velocity [rad/s] & $67.99(182.63)$ & $69.06(175.17)$ & $64.57(150.46)$ & $75.57(214.71)$ & $84.44(225.28)$ \\
\hline Acceleration $\left[\mathrm{m} / \mathrm{s}^{2}\right]$ & $0.00(1023.37)$ & $45.78(2112.64)$ & $152.02(3231.29)$ & $1744.46(5085.15)$ & $1289.19(3631.16)$ \\
\hline Angular Acceleration $\left[\mathrm{rad} / \mathrm{s}^{2}\right]$ & $0.00(26685.08)$ & $1239.99(53511.95)$ & $2381.37(79653.37)$ & $39388.53(125632.59)$ & $34341.13(100646.94)$ \\
\hline Max. translational sensitivity $[\mathrm{m} / \mathrm{rad}]$ & $0.16(0.31)$ & $0.18(0.38)$ & $0.18(0.34)$ & $0.17(0.32)$ & $0.17(0.34)$ \\
\hline Max. rotational sensitivity [ $\mathrm{rad} / \mathrm{rad}]$ & $4.56(14.83)$ & $3.68(9.22)$ & $3.67(6.13)$ & $2.93(6.35)$ & $3.58(8.18)$ \\
\hline Max. force sensitivity $[\mathrm{N} / \mathrm{N} \cdot \mathrm{m}]$ & $11.96(38.15)$ & $16.37(35.37)$ & $19.08(32.64)$ & $22.34(43.83)$ & $18.08(43.88)$ \\
\hline Max. torque sensitivity $[\mathrm{N} \cdot \mathrm{m} / \mathrm{N} \cdot \mathrm{m}]$ & $0.36(0.87)$ & $0.62(1.25)$ & $1.12(2.18)$ & $1.04(2.41)$ & $0.77(1.78)$ \\
\hline
\end{tabular}

The Octopod kinematics and the realized Octo have additionally been evaluated in the same workspace with rotations up to $\pm 30^{\circ}$ around all axes, while maintaining steps of $5^{\circ}$.

kinematics, with a minimum distance $l_{d}$, was found to be the optimal configuration among the 6-RSS kinematics that were studied.

3) Workspace, Worst Case Output Capabilities, and Sensitivities: Besides singularity avoidance, the criteria of output capabilities within the desired dexterous workspace have to be met. The workspace should dispose of a volume of $150 \mathrm{~mm} \times 150 \mathrm{~mm} \times 150 \mathrm{~mm}$, where rotations of $\pm 30^{\circ}$ around all axes are achievable. The volume of the workspace is depicted as a red cube in Fig. 5. The worst case output capabilities are analyzed for the selected kinematics. The kinematics are evaluated within the desired workspace volume with rotations restricted to $\pm 20^{\circ}$ around all axes to avoid Hunttype singularities. To evaluate the output capabilities and sensitivities, the algorithm by Ueberle [18] and the "polytope algorithm" in case of an actuation-redundant device are applied, respectively. The actuators, as used by Ergin and Peer [8], produce a nominal torque of $\tau_{i, \max , n}= \pm 2.5 \mathrm{~N} \cdot \mathrm{m}$, a stall torque of $\tau_{i, \max , s t}= \pm 34.2 \mathrm{~N} \cdot \mathrm{m}$, and a nominal velocity of $v_{i, \max }= \pm 51 \mathrm{rad} / \mathrm{s}$ after gearing. The Cartesian mass matrix is approximated as $\hat{\mathbf{M}}=\operatorname{diag}\left[\begin{array}{llllll}m & m & m & I_{x} & I_{y} & I_{z}\end{array}\right]$ with $m=0.17 \mathrm{~kg}, I_{x, y, z}=3 \times 10^{-4} \mathrm{kgm}^{2}$, and the similarity transformation to joint coordinates $\mathbf{M}_{q}(q)=\mathbf{J}(\mathbf{q})^{T} \hat{\mathbf{M}} \mathbf{J}(\mathbf{q})$. The minima and maxima of the worst case output capabilities as well as the maximum sensitivities of all kinematics within the desired workspace and under rotations around all axes are listed in Table III. Here, the Hunt-type kinematics shows the best maximum performance among the nonredundant kinematics in terms of worst case output capabilities. Interestingly, it is not systematically better than the other kinematics regarding the maximum sensitivity that quantifies worst case error amplification.

\section{Step 3: Redundant Extension of the Optimal Kinematics}

In the third step, we would like to further enhance the bestperforming kinematics, i.e., the Hunt-type kinematics. Of major concern are the rotations of the end effector that are restricted to $\pm 20^{\circ}$. Even in this range, the minimum continuous torque is only $0.05 \mathrm{~N} \cdot \mathrm{m}$, as presented in Table III. Redundancy can
TABLE IV

GII OptIMIZATION RESULTS

\begin{tabular}{lccc}
\hline \hline Kinematics & GII & $\sigma_{\min }$ & $\sigma_{\max }$ \\
\hline Hunt-type kinematics & 0.0006 & 0.0127 & 20.0052 \\
Octopod kinematics & 0.0080 & 0.2230 & 27.8186 \\
Octo kinematics & 0.0072 & 0.1910 & 26.6404 \\
\hline \hline
\end{tabular}

be employed to improve upon this issue and further enhance all output capabilities. Here, we use branch redundancy, instead of in-branch redundancy or kinematic redundancy, in order to avoid floating actuators or high mechanical complexity due to complicated kinematic chains. Moreover, in order to keep the number of required actuators low, we add only one pair of legs. Finally, we would like the isotropy of the device to be unimpaired by this modification and thus, aim for a symmetric shape of the kinematics. Thus, we propose an extension of the Hunttype kinematics to an Octopod kinematics with four symmetrical pairs of legs.

This should further decrease singularity occurrence, improve the GII as well as output capabilities. As shown in Table IV, the GII increases by a factor of 13 while the minimum singular value increases by a factor of 18 . Hence, the Octopod will show more isotropic behavior than the Hunt-type kinematics and is less likely to be trapped in singular configurations. Note that redundant kinematics such as the Octopod cannot be analyzed using the CuikSuite toolbox.

The desired dexterous workspace can be covered by the Octopod kinematics and its entire translatory workspace is depicted in blue in Fig. 5. The worst case output capabilities of the redundant Octopod kinematics are evaluated using rotations of $\pm 30^{\circ}$ around all axes. They are computed by employing the proposed "polytope algorithm." The actuator performance and Cartesian mass matrix was adopted from Ergin's device [8]. The worst case output capabilities of the Octopod kinematics in the plane of $z=0.30 \mathrm{~m}$ are depicted in Fig. 6. This is the same plane that was used for the evaluation of Ergin's device [8].

A force polytope that results from the evaluation of the Octopod kinematics is depicted in Fig. 7. The minimum force at 

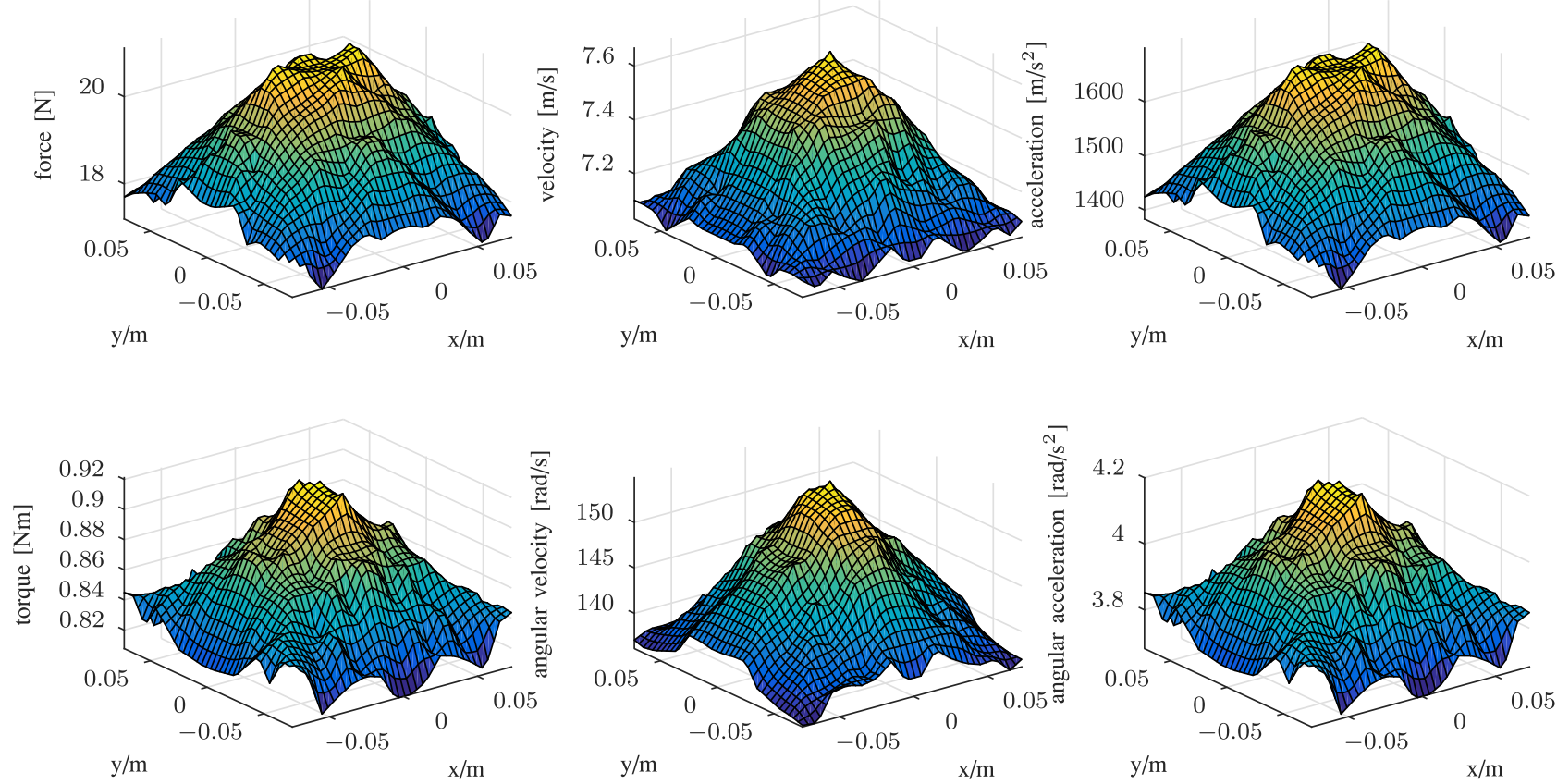

Fig. 6. Worst case output capabilities of the Octopod kinematics at $z=0.30 \mathrm{~m}$ with rotations of $\pm 30^{\circ}$ around all axes.

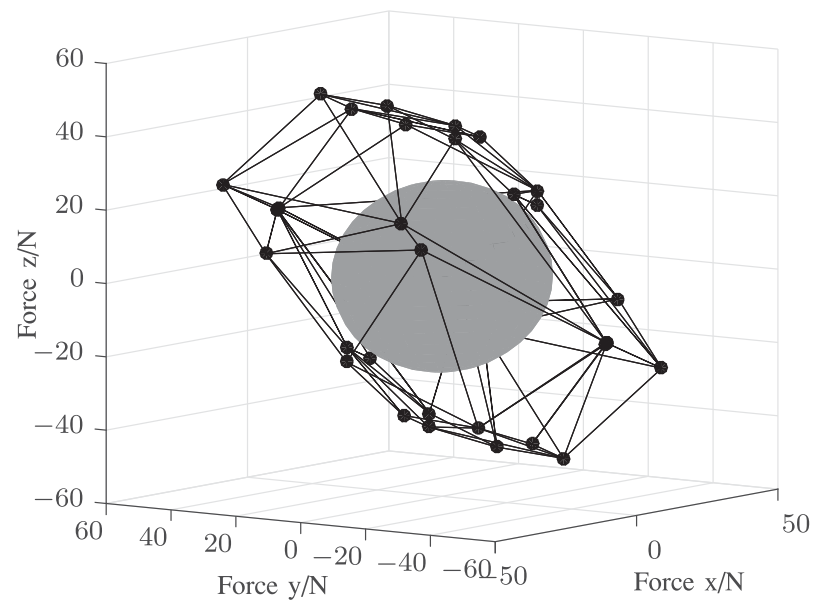

Fig. 7. Plot of a force polytope that was computed for the evaluation of the Octopod kinematics. The continuous force is represented by a grey sphere that is tangential to one of the surfaces of the polytope.

zero torque is represented by a sphere inside the polytope that is tangential to the surface closest to the origin.

The worst case output capabilities of the Octopod kinematics within the desired workspace, are listed in Table III. All nonredundant kinematics are outperformed by the Octopod kinematics in terms of minimum force, torque, and acceleration. The desired minima of acceleration and velocity can be achieved by the Octopod kinematics, also with rotations as high as $\pm 30^{\circ}$ around all axes. Moreover, the worst case maximum sensitivities of the redundant kinematics are also listed in Table III. The maximum force sensitivity increases, compared to the Hunt-type kinematics, whereas the other sensitivities show no significant change.
Even though the performance of the Octopod kinematics improves in many respects, control challenges arise: First of all, the resolution of the pseudoinverse is computationally intensive and restricts the maximum sampling time. Second, the redundancy generates internal tensions at the overconstrained end effector due to the geometric imperfections of a real system, measurement errors, or independent control of all actuators [27]. In order to address the issue of an overconstrained end effector, advanced control schemes have been proposed [27]-[29]. They augment standard proportional-integral (PD) or torque control in order to nullify internal forces [28], apply coordination motion control that takes into account parasitic forces due to tracking errors of neighboring kinematic chains [29], or propose a projection method for the elimination of contradicting decentralized control forces [27]. These advanced control methods were all evaluated using redundant parallel kinematics with two DOF. A thorough comparison of their performance with the MR-Octo offering six DOF is beyond the scope of this paper. However, we validated experimentally that the haptic interface can be controlled appropriately with a well-tuned PD controller. This means that internal forces exist, but they do not cause any vibration or other parasitic effects that impair the operation of the haptic interface. The implementation of this controller is presented in the following step.

\section{Step 4: Hardware Realization of the Octo Kinematics}

In the fourth and final step, a suboptimal solution of the Octopod kinematics is implemented. It is based on Ergin's device [8] and depicted in Fig. 8. Here, we refer to it as the "Octo." The Octo approximates the optimal parameter configuration in which angle $\gamma$ and length $l_{2}$ have not been reduced to zero to facilitate manufacturing. The kinematic parameters were set to 


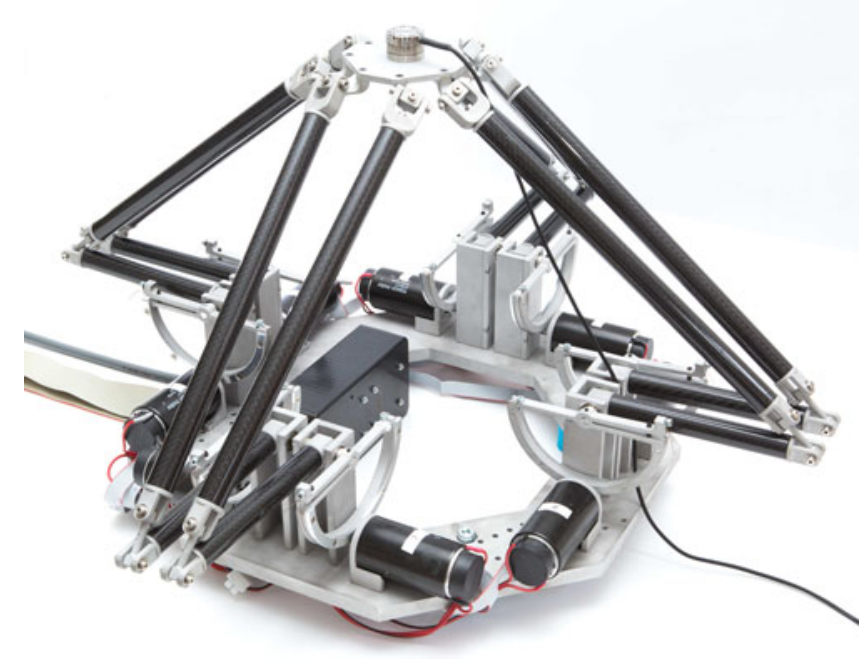

Fig. 8. Photograph of the realized Octo.

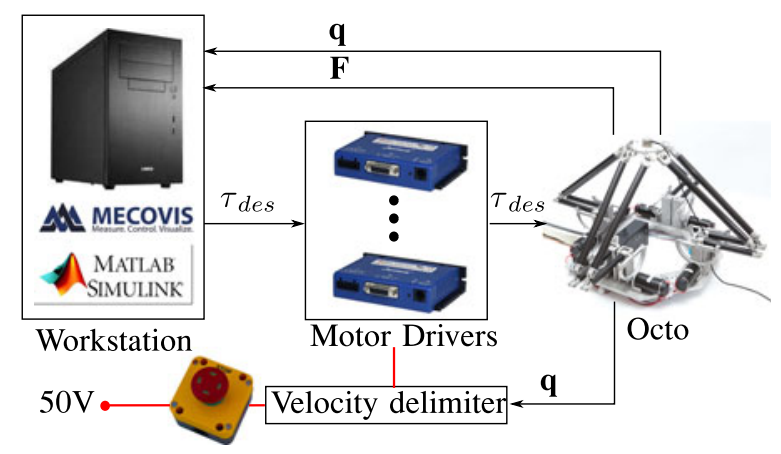

Fig. 9. Hardware setup and signal flow between the Octo and the workstation.

$l_{2}=25.6 \mathrm{~mm}, \beta=0^{\circ}, \gamma=13.18^{\circ}$, and $d=20 \mathrm{~mm}$. To have sufficient space for the motors, the radius of the base has been increased to $l_{0}=160 \mathrm{~mm}$. The resulting worst case output capabilities and GII are listed in Tables III and IV, respectively.

The haptic interface is actuated by eight Maxon RE $40 \mathrm{dc}$ motors. The motors are driven by Junus JSP-180-20 modules from Copley Controls, as depicted in Fig. 9. The Capstan gears have a ratio of 1:13. The six DOF force/torque sensor at the end effector is a Mini27 Titanium from ATI IA. The commands for the motors are generated via MATLAB/Simulink and executed by a Linux real-time kernel at a sampling rate of $1 \mathrm{kHz}$. Initially, MATLAB scripts and the MATLAB/Simulink Simmechanics toolbox have been tested for the implementation of the forward and inverse kinematics, but finally we decided for MotionGenesis Kane to solve the inverse kinematics problem, since its routines and solver are optimized for real-time applications. Inputs and outputs from the workstation to the haptic interface are managed via Mecovis I/O cards. The control of the haptic interface is governed by an admittance control scheme and the motors are controlled with a PD-controller at position level. This allows the control of the desired torque $\tau_{\text {des }}$ of the motors, based on their position $\mathbf{q}$ and the end effector wrench F. For the experiments, the gains of the proportional-integral-
TABLE V

EXPERIMENTAL AND SimUlative RESULTS OF Minimum Worst CASE OUTPUT CAPABILITIES OF THE OCTO

\begin{tabular}{lcc}
\hline \hline Output capability & Experiment & Simulation \\
\hline Continuous force $[\mathrm{N}]$ & 15.27 & 16.02 \\
Continuous torque $[\mathrm{N} \cdot \mathrm{m}]$ & 0.68 & 0.75 \\
Velocity $[\mathrm{m} / \mathrm{s}]$ & 1.12 & 2.93 \\
Angular velocity $[\mathrm{rad} / \mathrm{s}]$ & 24.02 & 84.44 \\
Acceleration $\left[\mathrm{m} / \mathrm{s}^{2}\right]$ & 459.52 & 1289.19 \\
Angular Acceleration $\left[\mathrm{rad} / \mathrm{s}^{2}\right]$ & 7051.21 & 34341.13 \\
\hline \hline
\end{tabular}

derivative (PID) controller are set to $K_{P}=110, K_{I}=0$, and $K_{D}=0.4$. The derivative of the error is additionally filtered with a low-pass filter using the forward Euler method with time constant $700 \mathrm{~ms}$.

The prototype was employed to validate our design criteria. First, the workspace was explored for singularity occurrence. Fichter's and Hunt's singularities are avoided within the dexterous workspace, as detailed in Table II. Furthermore, the dexterous workspace of the Octo can be enlarged to cover orientations of up to $30^{\circ}$ around all axes without the occurrence of singularities.

Second, the minima of the worst case output capabilities were verified. The end effector was positioned in the poses of its worst case output capabilities, as indicated by our theoretical analysis. Then, smoothed position step inputs were commanded in the respective directions. For each output capability five trials were executed and the mean was computed. Velocity and acceleration were not tested up to their maxima to avoid excessive wear on the structure. To validate worst case forces and torques, the end effector was blocked. The recorded values are listed in Table V and they show that our design criteria are met.

Last, the stiffness of the prototype was determined. The end effector position was gauged with a dial indicator that measured displacements along the major axes. This experiment was performed in 27 positions at zero rotations in the dexterous workspace. The positions included the center, the corners, as well as the center of each facet and of each edge. The minimum, maximum, and mean stiffness were $1.6 \mathrm{~N} / \mathrm{mm}$ along the $x$-axis, $7.9 \mathrm{~N} / \mathrm{mm}$ along the $z$-axis, and $4.7 \mathrm{~N} / \mathrm{mm}$, respectively. The minima are found on the upper facet of the dexterous workspace at $z=0.375 \mathrm{~m}$.

The experimental results are compared to commercial haptic interfaces in Table VI. It should be noted that the presented kinematics has been designed having its later application in an MR-compatible environment in mind. However, no MRcompatible haptic device that provides six DOF exists such that it could be taken as a reference. The kinematics chosen for comparison here cannot be rendered MR-compatible since either their main actuation principle cannot be changed to an MRcompatible one or they foresee actuators to be floating in space. Both would lead to motion artifacts in an MR scanner. Comparing the Octo to these devices, it is outperformed in terms of force capabilities by the Phantom Premium but its performance is comparable to the Delta.6 and Sigma.7. Moreover, the worst case torque of the Octo lies above the torque of any other device 
TABLE VI

COMPARISON OF THE DEVICE CAPABILITIES TO COMMERCIAL HAPTIC DEVICES

\begin{tabular}{|c|c|c|c|c|c|c|}
\hline \multirow[t]{2}{*}{ Output capability } & \multicolumn{6}{|c|}{ Device (value) } \\
\hline & Phantom Premium [30] & Maglev 200 [31] & Virtuose 6D [32] & Delta.6 [33] & Sigma.7 [34] & Octo \\
\hline Continuous force $[\mathrm{N}]$ & 37.5 & - & 3 & 20 & 20 & 15.27 \\
\hline Continuous torque $[\mathrm{N} \cdot \mathrm{m}]$ & 0.17 & - & 0.2 & 0.15 & 0.4 & 0.68 \\
\hline Max. translations $[\mathrm{m}]$ & $0.381 \times 0.267 \times 0.191$ & Sphere $\varnothing 0.024$ & $0.521 \times 0.370 \times 0.400$ & $\varnothing 0.40 \times 0.26$ & $\emptyset 0.19 \times 0.13$ & $0.15 \times 0.15 \times 0.15$ \\
\hline Max rotations $[\mathrm{deg}]$ & $297^{\circ} \times 260^{\circ} \times 335^{\circ}$ & $\pm 8^{\circ}$ & $270^{\circ} \times 120^{\circ} \times 250^{\circ}$ & $\pm 22^{\circ}$ & $235^{\circ} \times 140^{\circ} \times 200^{\circ}$ & $60^{\circ} \times 60^{\circ} \times 60^{\circ}$ \\
\hline Trans. stiffness $[\mathrm{N} / \mathrm{mm}]$ & 3.5 & $0.002-50$ & $\leq 1$ & - & - & $1.6-7.9$ \\
\hline
\end{tabular}

considered for comparison. The workspace of the Octo is only larger than the one of the Maglev 200. The translational stiffness of the Octo is again comparable to the Phantom Premium and outperforms the other devices as far as their performance is known.

\section{DISCUSSION}

In this study, we showed that the Hunt-type kinematics shows the best performance compared to the HEXA and Ergin's device, within the chosen workspace and in terms of singularity avoidance, achieving high isotropy as well as output capabilities. However, singularities remain when considering rotations among all axes. Thus, we suggested branch redundancy to reduce this problem. This also improved both output capabilities and isotropy. In order to calculate the worst case output capabilities as well as maximum sensitivities, the "polytope algorithm" has been applied. The computation of output capabilities was performed with a rotational resolution that guarantees reasonable computation times. This leads to a limited power to take conclusions on remaining singularities. Hence, the prototype has been employed to validate the absence of singularities and the worst case output capabilities. An evaluation showed that it has a lower stiffness than commercial devices such as the omega.3 from Force Dimension, which disposes of $14.5 \mathrm{~N} / \mathrm{mm}$. Apart from this, the results show a successful implementation of our design criteria. However, velocity and acceleration capabilities were calculated using a rigid body model that does not take into account practical limits resulting from the structural dynamics. Higher output capabilities could be achieved by a more rigid structure and a more advanced control scheme that reduces internal tensions in the overconstrained end effector.

\section{CONCLUSION}

In this study, we have introduced a novel Octopod kinematics. It has been derived from existing manipulators using a range of dynamic and kinematic performance criteria, such as GII, worst case output capabilities, sensitivity, and singularity occurrence. Typical direct kinematic singularities of 6 - $\underline{R} S S$ manipulators are avoided with this type of kinematics. It outperforms known haptic interfaces with nonfloating actuators and six DOF in terms of worst case force, torque, and acceleration. Future work will be directed toward the design of an MR-compatible haptic interface using the Octopod type of kinematics and improving its stiffness. The interface will then be employed to carry out human motor control studies in MR environments.

\section{ACKNOWLEDGMENT}

The authors would like to thank O. B. Nadal for his support with the CuikSuite toolbox.

\section{REFERENCES}

[1] S. Klare, A. Peer, and M. Buss, "Development of a three DOF MRcompatible haptic interface for pointing and reaching movements," in Haptics: Generating and Perceiving Tangible Sensations. Int. Conf., EuroHaptics 2010, Amsterdam, Jul. 8-10, 2010.

[2] S. Menon, H. Ganti, and O. Khatib, "Using haptic fMRI to enable interactive motor neuroimaging experiments," in Tracts in Advanced Robotics: Experimental Robotics. New York, NY, USA: Springer, 2014.

[3] F. Sergi, A. C. Erwin, and M. K. O'Malley, "Interaction control capabilities of an MR-compatible compliant actuator for wrist sensorimotor protocols during fMRI," IEEE/ASME Trans. Mechatronics, vol. 20, no. 6, pp. 2678-2690, Dec. 2015. [Online]. Available: http://ieeexplore. ieee.org/lpdocs/epic03/wrapper.htm?arnumber $=7046397$

[4] J.-P. Merlet, Parallel Robots, vol. 128. Springer Science \& Business Media, 2006.

[5] R. García-Rochín et al., "Second-order model for rotary traveling wave ultrasonic motors," Humanoids Robots (Humanoids), 2015 IEEE-RAS 15th Int. Conf., 2015.

[6] F. Pierrot, A. Fournier, and P. Dauchez, "Towards a fully-parallel 6 DOF robot for high-speed applications," in Proc. IEEE Int. Conf. Robot. Autom., 1991, pp. 1288-1293.

[7] Y. et al., "Design and analysis of a new six-DOF parallel haptic device with singularity-free task workspace," in Proc. ASME 2008 Int. Des. Eng. Tech. Conf. Comput. Inf. Eng. Conf., 2008, pp. 1131-1139.

[8] M. A. Ergin and A. Peer, "Development of a new 6 DOF parallel haptic interface for the rendering of elements and interior equipment in a car," in Proc. 2013 IEEE Ro-Man, 2013, pp. 238-244. [Online]. Available: http://ieeexplore.ieee.org/lpdocs/epic03/wrapper.htm?arnumber= 6628452

[9] J. H. Lee et al., "Singularity-free algorithms and design scheme for a new 6-DOF parallel haptic device," in Proc. IEEE Int. Conf. Robot. Autom., 2002, pp. 4229-4235. [Online]. Available: http://ieeexplore. ieee.org/lpdocs/epic03/wrapper.htm?arnumber $=1014419$

[10] de Vlugt et al., "A force-controlled planar haptic device for movement control analysis of the human arm," J. Neurosci. Methods, vol. 129, no. 2, pp. 151-168, 2003.

[11] P. Fischer and R. Daniel, "Specification and design of input devices for teleoperation," in Proc. IEEE Int. Conf. Robot. Autom., 1990, pp. 540-545.

[12] L. Stocco, S. E. Salcudean, and F. Sassani, "Fast constrained global minimax optimization of robot parameters," Robotica, vol. 16, no. 6, pp. 595-605, Jan. 1998.

[13] V. M. Hung and U. J. Na, "Optimal design of a new 6-DOF haptic device," in Proc. 11th Int. Conf. Control, Autom. Syst., 2011, pp. 849-852.

[14] A. Ahmad, K. Andersson, and U. Sellgren, "An optimization approach toward a robust design of six degrees of freedom haptic devices," J. Mech. Des., vol. 137, no. Apr., 2015, Art. no. 042301. [Online]. Available: http://mechanicaldesign.asmedigitalcollection.asme.org/ article.aspx?doi $=10.1115 / 1.4029514$ 
[15] V. Hayward and O. R. Astley, "Performance measures for haptic interfaces," in Proc. Int. Symp. Robot. Res., 1996, pp. 195-207.

[16] P. Bande, M. Seibt, E. Uhlmann, S. K. Saha, and P. V. M. Rao, "Kinematics analyses of Dodekapod," Mechanism Mach. Theory, vol. 40, no. 6, pp. 740-756, 2005.

[17] P. A. Voglewede and I. Ebert-Uphoff, "Measuring closeness to singularities for parallel manipulators," in Proc. 2004 IEEE Int. Conf. Robot. Autom., 2004, pp. 4539-4544.

[18] M.-W. Ueberle, "Design, Control, and Evaluation of a Family of Kinesthetic Haptic Interfaces," Ph.D. dissertation, Dept. Elect. Comput. Eng., Technische Universität München, Munich, Germany, 2006.

[19] J.-P. Merlet, "Singular configurations of parallel manipulators and Grassmann geometry," Int. J. Robot. Res., vol. 8, no. 5, pp. 45-56, 1989.

[20] M. Gouttefarde, D. Daney, and J. P. Merlet, "Interval-analysis-based determination of the wrench-feasible workspace of parallel cable-driven robots," IEEE Trans. Robot., vol. 27, no. 1, pp. 1-13, Feb. 2011.

[21] J. M. Porta, L. Ros, O. Bohigas, M. Manubens, C. Rosales, and L. Jaillet, "The CUIK suite," IEEE Robot. Autom. Mag., vol. 21, no. 3, pp. 105-114, Sep. 2014.

[22] L. J. Stocco, S. E. Salcudean, and F. Sassani, "On the use of scaling matrices for task-specific robot design," IEEE Trans. Robot. Autom., vol. 15 , no. 5, pp. 958-965, Oct. 1999.

[23] S. Krut, O. Company, and F. Pierrot, "Force performance indexes for parallel mechanisms with actuation redundancy, especially for parallel wiredriven manipulators," in Proc. 2004 IEEE/RSJ Int. Conf. Intell. Robots Syst., Sep. 2004, pp. 3936-3941.

[24] P. Cardou, S. Bouchard, and C. Gosselin, "Kinematic-sensitivity indices for dimensionally nonhomogeneous Jacobian matrices," IEEE Trans. Robot., vol. 26, no. 1, pp. 166-173, Feb. 2010.

[25] K. H. Hunt, "Structural kinematics of in-parallel-actuated robot-arms," J. Mech. Des., vol. 105, pp. 705-712, 1983.

[26] A. Wolf and M. Shoham, "Investigation of parallel manipulators using linear complex approximation," J. Mech. Des., vol. 125, no. 3, pp. 564$572,2003$.

[27] T. Hufnagel and A. Müller, "A projection method for the elimination of contradicting decentralized control forces in redundantly actuated PKM," IEEE Trans. Robot., vol. 28, no. 3, pp. 723-728, Jun. 2012.

[28] A. Mueller, "Problems in the control of redundantly actuated parallel manipulators caused by geometric imperfections," Meccanica, vol. 46, pp. 41-49, 2011.

[29] W. Shang, S. Cong, and Y. Ge, "Coordination motion control in the task space for parallel manipulators with actuation redundancy," IEEE Trans. Autom. Sci. Eng., vol. 10, no. 3, pp. 665-673, Jul. 2013.

[30] Phantom premium. [Online]. Available: http://www.geomagic.com/files/ 3413/6243/3387/EN-premium_15-6-specs.pdf

[31] Maglev 200. [Online]. Available: http://butterflyhaptics.com/products/ productspec.pdf

[32] Virtuose 6D Desktop. [Online]. Available: http://www.haption.com/site/ pdf/Datasheet_Virtuose_6DDesktop.pdf

[33] Delta.6. [Online]. Available: http://www.forcedimension.com/downloads/ specs/specsheet-delta.6.pdf

[34] Sigma.7. [Online]. Available: http://www.forcedimension.com/downloads/ specs/specsheet-sigma.7.pdf

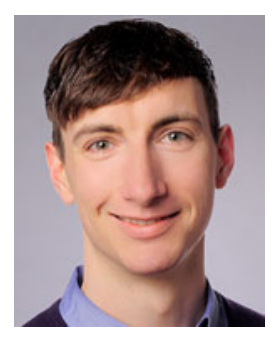

Markus Kühne received the Dipl.-Ing. degree in mechanical engineering from Technische Universität München, Munich, Germany; the diplôme d'ingénieur degree in robotics and embedded systems from École Nationale Supérieure de Techniques Avancées (ENSTA) ParisTech, Paris, France; and the master's degree in robotics from Université Pierre-et-MarieCurie (UPMC), Paris. He is currently working toward the Ph.D. degree in electrical engineering with the Biomimetic Robotics and Machine Learning Laboratory, Technische Universität München.

$\mathrm{He}$ is also affiliated with the Bristol Robotics Laboratory, University of the West of England, Bristol, U.K.

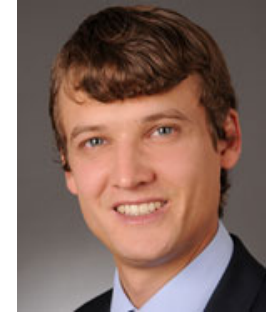

Johannes Potzy received the M.Sc. degree in control engineering and termo-fluid dynamics from the Karlsruher Institut für Technologie, Karlsruhe, Germany, and Technische Universität München, Munich, Germany, in 2014 $\mathrm{He}$ is currently working toward the Ph.D. degree with the Chair of Ergonomics, Technische Universität München, Munich.

During his studies, he was at the Royal Melbourne Institute, Melbourne, Australia, as part of a research project. After receiving the M.Sc. degree, he was a Control Engineer in the area of safety-related function development of Advanced Driver Assistance Systems at Ferchau Engineering $\mathrm{GmbH}$, Munich, Germany. He focuses on cooperative automatic driving in collaboration with Audi AG, Ingolstadt, Germany.

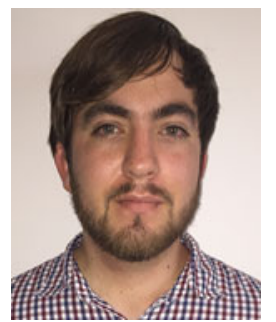

Roberto García-Rochín received the bachelor's degree (Hons.) in mechatronic engineering with specialization in robotics and automation from the Instituto Tecnologico de Culiacan, Culiacan, Mexico, in 2015. In 2016, he earned the Secretaria de Energia (SENER)-Consejo Nacional de Ciencia y Tecnologia (CONACyT) scholarship to study the master's degree in energy engineering with the Tecnologico de Monterrey, Monterrey, Mexico.

In 2014, he received a scholarship from the DELFIN program and worked at the Technische Universität Müenchen, Munich, Germany.

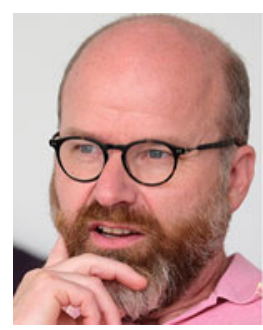

Patrick van der Smagt received the Ph.D. degree in mathematics and computer science from the University of Amsterdam, Amsterdam, Netherlands.

$\mathrm{He}$ is the Director of artificial intelligence research with the Volkswagen Group, Munich's Data Lab, Munich, Germany. His group focuses on unsupervised probabilistic deep learning, time series modeling, and robotics. He previously directed a lab as a Professor of machine learning and biomimetic robotics at the Technical University of Munich, Munich, and was the Head of bionics at the DLR, German Aerospace Centre, Oberpfaffenhofen, Germany. He is the Founding Chairman of a not-for-profit organization for assistive robotics for tetraplegics and a Co-Founder of various companies. He has published numerous papers and patents on machine learning, robotics, and motor control.

Mr. van der Smagt has received various awards, including the 2013 Helmholtz-Association Erwin Schrödinger Award, the 2014 King-Sun Fu Memorial Award, and the 2013 Harvard Medical School/MGH Martin Research Prize.

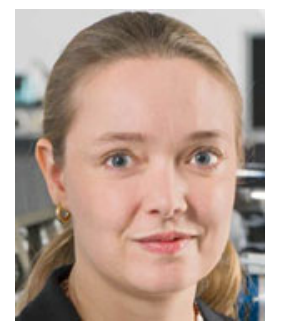

Angelika Peer received the diploma engineering degree in electrical engineering and information technology, and the doctor of engineering degree in electrical engineering from the Technische Universität München, Munich, Germany, in 2004 and 2008, respectively.

She is currently a Full Professor with the Bristol Robotics Laboratory, University of the West of England, Bristol, U.K. Before, she was a Senior Researcher and a Lecturer at the Institute of Automatic Control Engineering, Technische Universität München, and a Technical University of Munich - Institute of Advanced Studies (TUM-IAS) Junior Fellow at the Institute of Advanced Studies, Technische Universität München. Her research interests include robotics, haptics, teleoperation, human-human and human-robot interaction, as well as human motor control. 